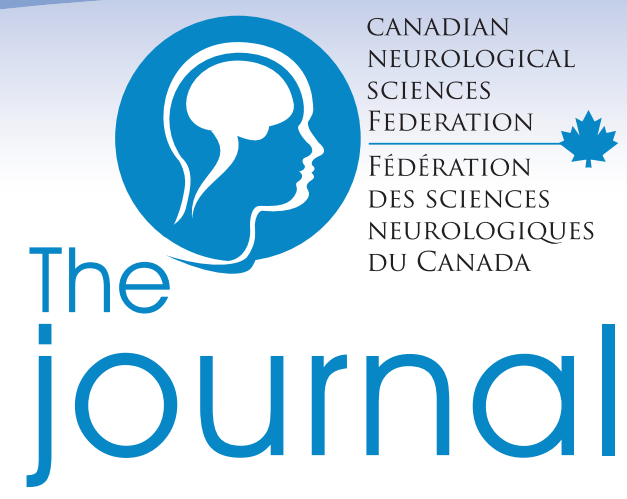

Canadian Journal of Neurological Sciences

Volume 43 (Supplement 3) June 2016

\title{
Dravet Syndrome: Addressing the Needs of Patients and Families
}

50th Annual Congress of the Canadian Neurological

Sciences Federation

Toronto, Canada, June 9th, 2015

Guest Editor: Charlotte Dravet (France)

\section{A Peer-reviewed SUPPLEMENT to}

The Canadian Journal of Neurological Sciences

Publication of this supplement was supported by BIOCODEX 


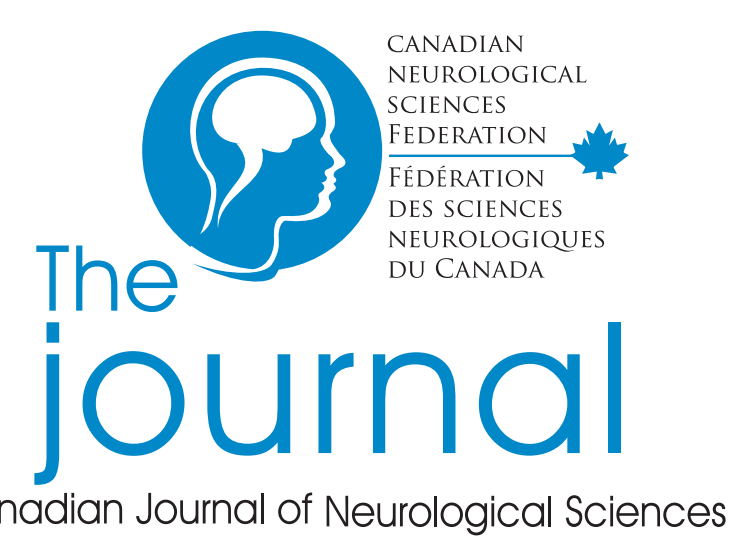

\section{Volume 43 / Number S3 / June 2016}

\section{INTRODUCTION}

S1 Dravet Syndrome: Addressing the Needs of Patients and Families: Introduction Charlotte Dravet

\section{ARTICLES}

S3 Dravet Syndrome: Diagnosis and Long-Term Course Mary B. Connolly

S9 Helping Families Cope with the Severe Stress of Dravet Syndrome Peter Camfield, Carol Camfield, Kathleen Nolan

S13 Treatment of Dravet Syndrome

Elaine C. Wirrell

\section{CONCLUSION}

S19 Dravet Syndrome: Addressing the Needs of Patients and Families: Conclusion Charlotte Dravet 


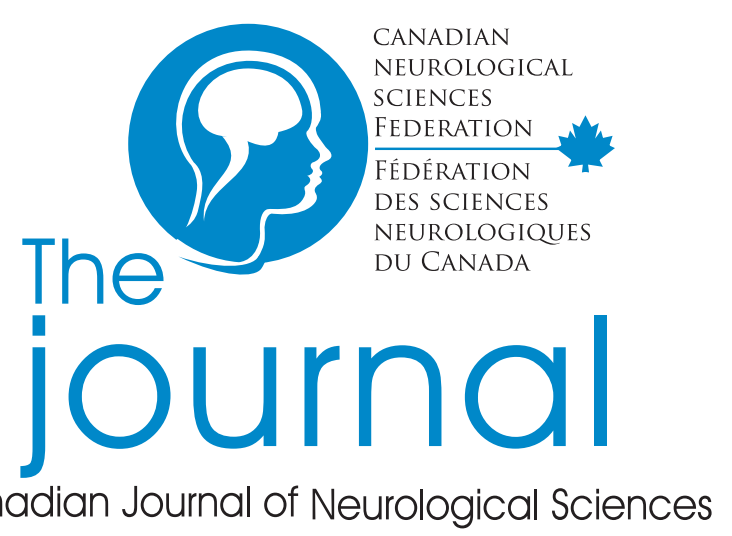

\section{Volume 43 / Number S3 / June 2016}

Editor-in-Chief/Rédactueur en chef

Robert Chen TORONTO, ON

\section{Associate Editors/Rédacteurs associés}

Etienne De Villers-Sidani MONTREAL, QC

Robert Hammond LONDON, ON

Hans Katzberg TORONTO, ON

Mahendranath Moharir TORONTO, ON

Tejas Sankar EDMONTON, AB

Manas Sharma LONDON, ON

Jeanne Teitelbaum MONTREAL, QC

\section{Past Editors/Anciens rédacteurs en chef}

G. Bryan Young LONDON, ON

Douglas W. Zochodne CALGARY, AB

James A. Sharpe TORONTO, ON

Robert G. Lee CALGARY, AB

Robert T. Ross WINNIPEG, MB (Emeritus Editor, Founding Editor)

\section{Editorial Board/Comité éditorial}

Jorge Burneo LONDON, ON

Richard Desbiens QUEBEC CITY, QC

David Fortin SHERBROOKE, QC

Mark Hamilton CALGARY, AB

Hans-Peter Hartung DUSSELDORF, GERMANY

Michael Hill CALGARY, ON

Alan C. Jackson WINNIPEG, MB

Daniel Keene otTAwA, ON

James Perry TORONTO, ON

Oksana Suchowersky EDMONTON, AB

Brian Toyota VAnCouver, BC

Brian Weinshenker ROCHESTER, MN, USA

Samuel Wiebe CALGARY, AB

Elaine Wirrell ROCHESTER, MN, USA

\section{Book Review/Critiques de livres Reflections/Reflets}

Andrew Kirk SASKatoon, SK

Editorial Review Board/Comité de lecture

Donald Brunet KINGSTON, ON
Jodie Burton CALGARY, AB

Lionel Carmant MONTREAL, QC

Colin Chalk MONTREAL, QC

K. Ming Chan edmonton, AB

Joseph Dooley HALIFAX, NS

Paolo Federico CALGARY, AB

Daryl Fourney SASKATOON, SK

Hannah Glass SAN FRANCISCO, CA, USA

Alan Goodridge ST. JOHNS, NL

Ian Grant HALIFAX, NS

Alan Gruberman OTTAWA, ON

John Hurlbert CALGARY, AB

Manouchehr Javidan VANCOUVER, BC

Patrick McDonald WINNIPEG, MB

Martin McKeown vancouver, BC

Joseph Megyesi LONDON, ON

Vivek Mehta EDMONTON, AB

Steven Miller TORONTO, ON

Neelan Pillay CALGARY, AB

Christopher Power EDMONTON, AB

Alex Rajput SASKATOON, SK

Jean Raymond MONTREAL, QC

Gary Redekop vancouver, BC

Harvey Sarnet CALGARY, AB

John Stewart VANCOUVER, BC

Jeanne Teitelbaum MONTREAL, QC

Eve Tsai OTTAwA, ON

Shannon Venance LONDON, ON

Matt Whetley EDMONTON, AB

Jerome Yager EDMONTON, AB

\section{Journal Staff/Effectif du Journal}

Dan Morin CALGARY, AB

Chief Executive Officer

\section{The official journal of: / La Revue officielle de:}

The Canadian Neurological Society La Société Canadienne de Neurologie

The Canadian Neurosurgical Society

La Société Canadienne de Neurochirurgie

The Canadian Society of Clinical Neurophysiologists La Société Canadienne de Neurophysiologie Clinique
The Canadian Association of Child Neurology L' Association Canadienne de Neurologie Pédiatrique

The permanent secretariat for the four societies and the Canadian Neurological Sciences Federation is at:

Le sécretariat des quatre associations et de la Fédération des sciences neurologiques du Canada est situe en permanence à :

7015 Macleod Trail SW, Suite 709 Calgary, Alberta, Canada T2H 2K6 CNSF (403) 229-9544 / CJNS (403) 229-9575 Fax (403) 229-1661

The Canadian Journal of Neurological Sciences is published bi-monthly. The annual subscription rate for Individuals (electronic) is $£ 117 / \$ 193$. The annual subscription rate for Institutions (electronic) is $£ 156 / \$ 259$. See <www.journals. cambridge.org/cjn $>$ for full details including taxes; e-mail: subscriptions_ newyork@cambridge.org. The Canadian Journal of Neurological Sciences is included in the Cambridge Journals Online service, which can be accessed at www.journals.cambridge.org. For information on other Cambridge titles, visit www.cambridge.org. For advertising rates contact M. J. Mrvica Associates, 2 West Taunton Avenue, Berlin, NJ 08009; Phone: 856-768-9360; Fax: 856-7530064; Email: mjmrvica@mrvica.com.

Le Journal Canadien des Sciences Neuorlogiques est publié tous les deux mois. Le prix d'abonnement annuel pour les individus (électronique) est $111 £ / 184 \$$. Le prix d'abonnement annuel pour les établissements (électronique) est 125£/207\$. Veuillez consulter <www.journals.cambridge.org/cjn $>$ pour tous les détails, y compris les taxes; email: subscriptions_newyork@cambridge.org. Le Journal canadien des sciences neurologiques est inclus dans le service Cambridge Journals Online, accessible à www.journals.cambridge.org. Pour plus

d'informations sur les titres disponible chez Cambridge, veuillez consulter www. cambridge.org. Pour les tarifs de publicité, contacter M. J. Mrvica Associates, 2 West Taunton Avenue, Berlin, NJ 08009; Téléphone: (1)856-768-9360; Email: mjmrvica@mrvica.com.

This journal is indexed by / Cette revue est indexée par:

Adis International, ArticleFirst, BIOBASE, BioLAb, BiolSci, BIOSIS Prev, Centre National de la Recherche Scientifique, CSA, CurAb, CurCont, De Gruyter Saur, E-psyche, EBSCO, Elsevier, EMBASE, FRANCIS, IBZ, Internationale Bibliographie der Rezensionen Geistes-und Sozialwissenschaftlicher Literatur, MEDLINE, MetaPress, National Library of Medicine, OCLC, PE\&ON, Personal Alert, PsycFIRST, PsycINFO, PubMed, Reac, RefZh, SCI, SCOPUS, Thomson Reuters, TOCprem, VINITI RAN, Web of Science.

ISSN: 0317-1671

EISSN: 2057-0155

COPYRIGHT $\odot 2016$ by THE CANADIAN JOURNAL OF NEUROLOGICAL SCIENCES INC. All rights reserved. No part of this publication may be reproduced, in any form or by any means, electronic, photocopying, or otherwise, without permission in writing from Cambridge University Press. Policies, request forms and contacts are available at: http://www.cambridge.org/about-us/rights-permissions. Permission to copy (for users in the U.S.A.) is available from Copyright Clearance Center: http://www.copyright.com, email: info@ copyright.com.

COPYRIGHT @ 2016 du THE CANADIAN JOURNAL OF NEUROLOGICAL SCIENCES INC. Tous droits réservés.

Aucune partie de cette publication ne peut être reproduite, sous quelque forme ou par quelque procédé que ce soit, électronique ou autre, y compris la photocopie, sans l'accord écrit de Cambridge University Press. Les politiques, les formulaires de demande et les contacts sont disponibles à: http://www.cambridge.org/aboutus/rights-permissions. La permission de copier (pour les utilisateurs aux ÉtatsUnis) est disponible auprès Copyright Clearance Center: http://www.copyright. com, email: info@copyright.com. 